\title{
Erratum
}

\section{Erratum to: The Holst Action by the Spectral Action Principle}

\author{
Frank Pfäffle $^{1}$, Christoph A. Stephan ${ }^{2,3}$ \\ 1 Mathematisches Institut, Georg-August Universität Göttingen, Bunsenstraße 3-5, \\ Göttingen, Germany. E-mail: pfaeffle@uni-math.gwdg.de \\ 2 Fachbereich Mathematik, Universität Hamburg, Bundesstraße 55, Hamburg, Germany. \\ E-mail: christophstephan@gmx.de \\ 3 Institut für Mathematik, Universität Potsdam, Am Neuen Palais 10, Potsdam, Germany
}

Received: 31 March 2012 / Accepted: 4 May 2012

Published online: 30 May 2012 - (C) Springer-Verlag 2012

Commun. Math. Phys. 307, no. 1, 261-273 (2011)

There is a sign mistake in the proof of Proposition 2.3: in the equation before (9) one should have $-12 T \wedge V^{b}=12\left\langle T, * V^{b}\right\rangle_{3}$ dvol for a 3 -form $T$ and a vector field $V$ on an oriented 4-manifold.

As a consequence Proposition 2.3 and our main result in Theorem 2.4 get changed:

Proposition 2.3. For any orthogonal connection $\nabla$ one finds

$$
C_{H}=6 d T-12\left\langle T, * V^{b}\right\rangle_{3} \mathrm{dvol}-\frac{1}{2}\left(\left\|S_{+}\right\|^{2}-\left\|S_{-}\right\|^{2}\right) \mathrm{dvol} .
$$

Hence in Eq. (10) the density of the Holst action reads

$$
\begin{aligned}
\rho_{\gamma}^{\nabla} \mathrm{dvol}= & \left(R^{g}+6 \operatorname{div}^{g}(V)-6|V|^{2}-\|T\|^{2}+\frac{12}{\gamma}\left\langle T, * V^{\mathrm{b}}\right\rangle_{3}\right) \mathrm{dvol}-\frac{6}{\gamma} d T \\
& +\left(\frac{1}{2}\left(1+\frac{1}{\gamma}\right)\left\|S^{+}\right\|^{2}+\frac{1}{2}\left(1-\frac{1}{\gamma}\right)\left\|S^{-}\right\|^{2}\right) \mathrm{dvol} .
\end{aligned}
$$

And we have to modify the main result:

Theorem 2.4. Let $M$ be a compact Riemannian 4-manifold with spin structure. For a 3-form $T$ and a vector field $V$ consider the orthogonal connection $\nabla_{X} Y=\nabla_{X}^{g} Y+$ $T(X, Y, \cdot)^{\sharp}+g(X, Y) V-g(V, Y) X$. Let D denote the Dirac operator induced by $\nabla$, and consider the restriction $P_{L} D^{*} D P_{L}$ to the left-handed spinors. Then, for the term $\beta_{2}$ from the heat kernel expansion (14) we have

$$
\beta_{2} \mathrm{dvol}=-\frac{1}{6} \rho_{\gamma}^{\bar{\nabla}} \mathrm{dvol}
$$

The online version of the original article can be found under doi:10.1007/s00220-011-1303-0. 
for the orthogonal connection $\bar{\nabla}$ given by $\bar{\nabla}_{X} Y=\nabla_{X}^{g} Y+3 T(X, Y, \cdot)^{\sharp}+3 g(X, Y) V-$ $3 g(V, Y) X$ and for the value $\gamma=1$ of the Barbero-Immirzi parameter.

The first difference to the previous version of the theorem is that on the right-hand side of (15) there is no term besides the Holst density. The other difference is that the connection $\bar{\nabla}$ induces the spinor connection $\widetilde{\nabla}$ appearing in the Lichnerowicz formula in Theorem 3.3. Then Corollary 3.5 remains unchanged.

We think that the results are now simpler and even more natural than in the published version.

Communicated by A. Connes 